\title{
BMJ Open Protocol for a scoping review exploring the use of patient-reported outcomes in adult social care
}

\author{
Sarah E Hughes (D) , ${ }^{1,2,3}$ Olalekan Lee Aiyegbusi, ${ }^{1,2,3,4,5}$ Daniel S Lasserson, ${ }^{6}$ \\ Philip Collis, ${ }^{1}$ Samantha Cruz Rivera (D) , ${ }^{1,2,7}$ Christel McMullan (D) ,,2 \\ Grace M Turner, ${ }^{1,2}$ Jon Glasby, ${ }^{8}$ Melanie Calvert (D) ${ }^{1,2,3,4,7,9}$
}

To cite: Hughes SE, Aiyegbusi OL, Lasserson DS, et al. Protocol for a scoping review exploring the use of patient-reported outcomes in adult social care. BMJ Open 2021;11:e045206. doi:10.1136/ bmjopen-2020-045206

- Prepublication history and additional supplemental material for this paper are available online. To view these files, please visit the journal online (http://dx.doi.org/10.1136/ bmjopen-2020-045206).

Received 24 September 2020 Revised 02 February 2021 Accepted 12 March 2021

Check for updates

(C) Author(s) (or their employer(s)) 2021. Re-use permitted under CC BY. Published by BMJ.

For numbered affiliations see end of article.

Correspondence to

Sarah E Hughes;

s.e.hughes@bham.ac.uk

\section{ABSTRACT}

Introduction Patient-reported outcomes (PROs) are

measures of a person's own views of their health, functioning and quality of life. They are typically assessed using validated, self-completed questionnaires known as patient-reported outcome measures (PROMs). PROMs are used in healthcare settings to support care planning, clinical decision-making, patient-practitioner communication and quality improvement. PROMs have a potential role in the delivery of social care where people often have multiple and complex long-term health conditions. However, the use of PROMs in this context is currently unclear. The objective of this scoping review is to explore the evidence relating to the use of PROMs in adult social care.

Methods and analyses The electronic databases Medline (Ovid), PsychInfo (Ovid), ASSIA (ProQuest), Social Care Online (SCIE), Web of Science and EMBASE (Ovid) were searched on 29 September 2020 to identify eligible studies and other publically available documents published since 2010. A grey literature search and hand searching of citations and reference lists of the included studies will also be undertaken. No restrictions on study design or language of publication will be applied. Screening and data extraction will be completed independently by two reviewers. Quality appraisal of the included documents will use the Critical Appraisal Skills Programme and AACODS (Authority, Accuracy, Coverage, Objectivity, Date, Significance) checklists. A customised data charting table will be used for data extraction, with analysis of qualitative data using the framework method. The review findings will be presented as tables and in a narrative summary.

Ethics and dissemination Ethical review is not required as scoping reviews are a form of secondary data analysis that synthesise data from publically available sources. Review findings will be shared with service users and other relevant stakeholders and disseminated through a peer-reviewed publication and conference presentations. This protocol is registered on the Open Science Framework (www.osf.io).

\section{INTRODUCTION}

People with care and support needs often live with multiple and complex, long-term health conditions. They are likely to experience greater levels of disability and have complex
Strengths and limitations of this study

- This scoping review will follow the Preferred Reporting Items for Systematic Reviews and Metaanalyses (PRISMA) guidelines for the conduct of scoping reviews, ensuring transparency and rigour.

- Service user and community involvement in the design and conduct of the review will ensure that the findings are supported by stakeholder experiences, further helping to identify evidence gaps and research priorities.

- An appraisal of the methodological quality of studies is not a requirement of a scoping review; however, a quality appraisal of the included research articles will be conducted to gather insights into the types, sources and quality of the evidence around the use of patient-reported outcomes in adult social care.

care and support needs. ${ }^{23}$ An understanding of a person's own views about their health can help ensure care and support is provided in a way that is holistic, person-centred and responsive. Such insights are particularly relevant in the UK where a fragmented health and social care system make the coordination of care challenging. ${ }^{4}$

Individuals' perspectives about their health are most useful to care providers when these first-hand accounts are both relevant and reliably measured..$^{5}$ A patient-reported outcome (PRO) is 'a measurement of any aspect of a patient's health that comes directly from the patient without the interpretation of the patient's responses by anyone else'. ${ }^{6}$ PROs include symptom severity of the impact of a disease or its treatment on a patient's healthrelated quality of life (including functioning and social and emotional well-being) ${ }^{7}$ PROs are typically measured using self-report questionnaires known as patient-reported outcome measures (PROMs).

PROs are implemented widely across different levels of healthcare. ${ }^{8}$ At an individual 
level, PROs may be used to inform decision-making around treatment, monitor disease symptoms and treatment adherence and facilitate communication between the patient and their clinical team. At a systems level, PROs may be used to support benchmarking including service evaluation and commissioning of services, inform policymaking and support quality improvement initiatives. PROs are used as endpoints within clinical trials, support regulatory approval of new medicinal products and devices and inform clinical guidelines and health policy. ${ }^{5} 910$ PROs are valued due to their capability to ensure that the patient's unique perspective is represented in the measurement of health and when capturing the effectiveness of healthcare interventions.

Despite their established use in healthcare, the role of PROs and PROMs in social care is less clear. Published reviews have tended to study PROMs within a specific context, health condition or population. For example, reviews have explored the use of PROMs in primary care and investigated stakeholders' perspectives regarding the use of PROMs with frail, older adults living at home. ${ }^{11} 12$ Reviews have also been conducted that focus on the use of PROMs in end-of-life care and in the care of people with dementia and other long-term conditions. ${ }^{13-16}$ To date, a review focussing on the use of PROMs in the broader context of adult social care has not been identified.

The Care Act (2014) places a duty on local authorities to ensure that the well-being of individuals is placed at the heart of care and support. ${ }^{17}$ Commensurately, in social care, PROMs have focused on measuring the wider determinants of well-being (eg, personal dignity, control by an individual, participation in work or training and mental, physical and emotional health) and social care-related quality of life ${ }^{18}$ However, it has been suggested that health, as a component of well-being, may be more appropriately measured with health-related PROMs as a complement existing social care measures. ${ }^{19}{ }^{20}$ A better understanding of how health and social care-related PROMs are used in the delivery of care and support, by whom, and the barriers and facilitators to their utilisation may be considered increasingly relevant as decision-making around care and support becomes more person focussed and data driven. ${ }^{21}$ A comprehensive understanding of PROs may be particularly pertinent as health, care and support services move towards greater integration. ${ }^{22}$ Clarification on PROM use is especially timely in light of the COVID-19 pandemic, which has resulted in the increased use of telemedicine, virtual consultations and remote assessment and monitoring. ${ }^{23}{ }^{24}$ As such, PROMs have the potential to help people with care and support needs express their views about their health (including symptoms, functioning and quality of life) and well-being. ${ }^{25}$

Consistent with the remit of scoping reviews as a form of knowledge synthesis, the aim of this scoping review is to explore the existing evidence to understand the current state of knowledge on the use of PROs and PROMs in adult social care. To achieve this aim, the specific review objectives are: (1) to identify PROMs used by people to meet their own direct care and support needs, (2) to characterise how PROMs have been used in this context; and to chart the evidence relating to (3) their effectiveness, (4) barriers and facilitators to their implementation and (5) stakeholder views on their use. A subanalysis to explore the use of PROMs in the delivery of integrated health and social care will also be undertaken. ${ }^{26}$

\section{A shared language for PROs}

The term 'patient-reported outcome' is a reflection of PROs' origins in medicine and healthcare. However, in the context of social care people with care and support needs rarely identify as patients, preferring to identify as service users or citizens. ${ }^{27}$ In this review on the use of PROs within adult social care, the terms 'person-reported outcome' and 'person-reported outcome measure' will be used.

\section{METHODS AND ANALYSIS}

\section{Protocol design, registration and reporting}

Scoping reviews are conducted to understand the existing research on a given topic and often serve as a precursor to systematic reviews. ${ }^{28}$ Specifically, scoping reviews seek to identify and map the available evidence and, in so doing, identify knowledge gaps and directions for future research. ${ }^{29}$ Frequently, scoping reviews are complemented by stakeholder consultation exercises to ensure a holistic understanding of the topic that is informed by both the published evidence and lived experience. ${ }^{30}$ This scoping review will use the methodology proposed by the Joanna Briggs Institute, supported by the original scoping framework proposed by Arksey and O'Malley and Peters et al. ${ }^{31}$ The Preferred Reporting Items for Systematic Reviews and Meta-analyses (PRISMA) guidance for protocol development and scoping reviews and Joanna Briggs Institute (JBI) reporting checklists were consulted when preparing this protocol. ${ }^{31-33}$ This review protocol is registered online with the Open Science Framework ( www.osf.io).

\section{Eligibility criteria}

Participants

The review will consider sources of evidence that report on adults ( $\geq 18$ years of age) who are direct users of social care services or receive integrated health and social care. No limitations with regards to an individual's health condition(s) will be applied.

\section{Concept}

Sources of evidence that provide information on any PROM used in integrated or social care will be considered for inclusion in the review. Self-report instruments that are proxy-reported and carer-reported measures will be excluded.

\section{Context}

Sources of evidence reporting on a social care or integrated care intervention will be considered. An intervention may 
be defined as an 'action where someone gets involved to improve a situation or prevent it getting worse' that is undertaken with, or on behalf of, a service user to promote independence, provide support, prevent harm and enable them to live their lives in accordance with their own wishes and beliefs. ${ }^{34}{ }^{35}$ Integrated care refers to coordinated health and social care that is planned and organised around the needs and preferences of the individual, their carers and family. ${ }^{22} 34$ The review will exclude studies and other evidence sources set in primary and secondary care contexts.

\section{Types of sources}

This scoping review will consider all sources of published evidence on the use of PROs and PROMs in adult social care. Sources of evidence will include primary research studies, websites, guidelines, government reports and policy documents. Expert opinions, editorials and studies reporting secondary data analysis (ie, systematic reviews) will also be included.

\section{Information sources}

The searches will be conducted in three stages: (1) electronic database searching, (2) grey literature searching and (3) hand searching the reference lists and citations of included sources to identify further studies for inclusion. Electronic database selection will be based on recommendations for optimal database searching for sensitivity. ${ }^{36}$

The electronic databases Medline (Ovid), Embase (Ovid), PsychInfo (Ovid), HMIC (Ovid), Social Care Online (SCIE), ASSIA (Pro Quest) and Web of Science (Pro Quest) were searched on 29 September 2020 for the period 2010 to present date, with no language limitation. Sources published in languages other than English will be translated. Grey literature databases, Google and websites of government and third sector organisations will be searched to locate relevant sources. Hand searching of all of the included sources' reference lists will also be undertaken. Grey literature will be defined as "work that is produced on all levels of government, academia, business and industry in print and electronic formats but which is not controlled by commercial publishers. ${ }^{37}$ A 10 -year date limit on searches was considered appropriate for locating relevant sources, reflecting the increased use of PROMs over the last decade, the introduction of the UK National PROMs programme, publication of the FDA's guidance on PROMs and the UK government's National Health Service (NHS) white paper outlining government's vision for greater integration of health and social care. ${ }^{638} 39$

\section{Search strategy}

The Medline search strategy will be developed and then adapted for use with other databases. The search strategy will combine MeSH terms (and relevant synonyms) and the Boolean operators "AND" and "OR" for search strings. Four concept clusters will be included: (1) the concept of interest (ie, PROs and PROMs), (2) the population of adults who use social care or integrated care services
(3) contextual factors including type of integrated care/ social care intervention and (4) PROM implementation (eg, barriers and facilitators). Terms were developed by the review team with support from an information science specialist. Service user partner and coinvestigator (PC) was consulted to identify specific search terms related to integrated care and social care. The grey literature search will take place after searches of the electronic databases have been completed to enable to study team to immerse themselves in the literature and come to better understand the evidence base. Hand searching of citations and reference lists of included documents will be undertaken at each stage of the search process. The Medline search strategy is presented in online supplemental file 1.

\section{Selection of sources of evidence}

A team of reviewers (SEH, GT, CM, SCR) will conduct title/abstract and full-text screening of all records against prespecified eligibility criteria to ascertain suitability for inclusion in the review. All records will be screened independently by a minimum of two reviewers. Disagreements between reviewers will be resolved through discussion. If necessary, a third reviewer will be involved if consensus cannot be reached. The eligibility criteria will be pretested on a sample of abstracts to ensure that evidence sources discussing the use of PROs and PROMs in integrated/ social care are captured. Records identified from electronic databases, the grey literature and hand searching will be imported to Endnote V.9.3.3 (www.endnote.com) reference management software, and duplicate records will be removed. All screening will be conducted within Endnote. Any retrieved documents deemed to meet the inclusion criteria will go forward for full-text review. For articles excluded during full-text screening, a reason for exclusion will be documented. Disagreements on article inclusion will be resolved through discussion by members of the review team until consensus is achieved. The study selection process will be documented in a PRISMA flow diagram.

\section{Critical appraisal of individual sources of evidence}

Assessment of the methodological quality of included studies is not a requirement of scoping reviews. ${ }^{31}$ The decision to undertake a quality appraisal will be governed by the number and type of sources identified by the searches. If feasible, a formal quality assessment will be undertaken using the Critical Appraisal Skills Programme checklists and the AACODS (Authority, Accuracy, Coverage, Objectivity, Date, Significance) checklist for quality appraisal of the grey literature. ${ }^{40}{ }^{41}$ Appraisals will be undertaken to characterise the overall quality of the evidence base and no study will be excluded on the basis of its methodological quality. Two reviewers will independently appraise studies for quality. Findings will be collated by study type and presented in tabular format along with a textual description of findings. To ensure transparency of reporting, interrater agreement and reliability will be 
determined by calculating an intraclass coefficient (ICC) statistic, Cohen's $\kappa$ and percentage agreement. ${ }^{42} 43$

\section{Data charting process}

Data extraction, termed data charting in scoping reviews, aims to provide a 'logical and descriptive summary of results that align with the review questions'. ${ }^{31}$ A standardised template will be developed in Excel and piloted prior to commencing data charting. Two reviewers will extract key characteristics of the data source and information relevant to answering the review questions independently. Discrepancies will be resolved through discussion. As a scoping review is an iterative process, the data extraction form will be refined and updated throughout the course of the review and in response to the emergent findings.

\section{Data items}

The data items to be extracted for each data source comprise: (1) the publication title, (2) date of publication, (3) authors, (4) country (location of study), (5) study aims and objectives, (6) study design, (7) study setting, (8) whether the intervention is an integrated care intervention, (9) study population, (10) study data collection method, (11) data analysis method. Information on community and user involvement, study outcome(s), process and system-level outcomes in response to the PROM intervention and barriers/enablers to implementation will also be extracted. PROM-specific information will be charted separately and include a list of studies using each PROM and, if available, a description of the PROMs' characteristics, including target population, construct domains, number of items/subscales, mode of administration, recall period, response options, range of scores, scoring, language of the original publication and available translations.

\section{Data summary}

A qualitative content analysis in scoping reviews is generally descriptive in nature. ${ }^{31}$ Following data charting, findings from the included sources will be collated with analysis of common themes relating to the aims of the review. Given the anticipated diversity of the included data sources, the framework method, using NVivo (V.1.3) qualitative data analysis software and Microsoft Excel spreadsheets and data charting, will be used to capture key themes. ${ }^{445}$ The framework method sits within the family of methods described as thematic analysis or content analysis. Its hallmark is the systematic construction of a matrix that facilitates comparison across and within cases (ie, data sources) to produce a thematic interpretation of the data. The framework method follows a structured format, whereby the reviewers will first become familiar with the data source. The data will be reviewed line-byline and coded by applying a paraphrase or label. Coding will include a number of a priori deductive codes selected for their relevance to the aims of this scoping review to chart the evidence of PROM use in adult social care. The reviewer will also deploy inductive coding by remaining open to the coding of new and unexpected concepts that may be emergent in the data. The first few data sources will be coded independently by two reviewers. Through consensus discussions, the reviewers will agree on a set of codes to be applied to all subsequent sources. Codes will be grouped into categories to create an analytical framework that will be used for indexing the remaining data sources. Finally, the coded data will be charted into a matrix using a spreadsheet. The characteristics of and differences between the data will be identified and a description of relationships between categories will be developed. Results will be presented using tables and diagrams accompanied by a narrative summary. ${ }^{31}$

\section{Piloting/calibration exercise}

All phases of the scoping review will be pretested. The JBI recommended procedure for pilot testing will be used. ${ }^{31}$ For screening, the titles and abstracts of 25 randomly selected articles will be screened by the review team, applying the a priori review eligibility criteria. Screening will commence once inter-rater agreement of $75 \%$ or greater is achieved. Inter-rater agreement that is less than $75 \%$ will prompt the study team to review and refine the inclusion criteria. This process of pilot testing will continue iteratively until the $75 \%$ agreement threshold is met.

The data charting template will be piloted by two reviewers to ensure the capture of data relevant to the aims and objective of the review. As the review progresses, the reviewers may wish to extract additional information of relevance to the review objectives; therefore, further revisions to the data chart template may be made iteratively as the review progresses. ${ }^{31}$

\section{Changes to the protocol}

The exploratory nature and breadth of a scoping review mean that changes to the protocol may be required as new information becomes available and as the review progresses. Any protocol deviations or refinements, together with a rationale for these changes, will be documented clearly in the review report.

\section{Service user and public involvement}

This scoping review was conceived and developed with community and user involvement. As coinvestigator and a person with lived experience, PC contributed to the development of the search strategies, the study protocol, consensus discussions and will review all studyrelated documents. A lay summary to facilitate dissemination of the review findings will be coproduced. In addition, this work will be discussed with the NIHR ARC West Midlands Long Term Conditions Theme 1 Patient and Public Involvement and Engagement Stakeholder Advisory Group. Meetings will be held on a quarterly basis throughout the review process. The Guidance for Reporting Involvement of Patients and the Public-2 short 
form checklist will be used to document service user and public involvement. ${ }^{46}$

\section{Ethics and dissemination}

Ethical approval is not required as this review is a retrospective review of publicly available evidence sources. The review findings will be disseminated via publication in a peer-reviewed journal, symposia and conference presentations. A dissemination strategy to ensure the review findings reach relevant stakeholders will be coproduced in partnership with members of the review team who are experts by experience.

\section{DISCUSSION}

PROs can provide key data that enable health and social care practitioners to understand better a person's views about their health and well-being, allowing them to provide effective, efficient and compassionate care. This scoping review will be the first to provide an overview of the evidence around the use of PROs with adults who use social care services. Given the disparate nature of the evidence, the use of a scoping review methodology is appropriate as it enables the review to evolve in an iterative manner that is responsive to the evidence.

\section{Author affiliations}

${ }^{1}$ Centre for Patient Reported Outcomes Research (CPROR), University of Birmingham, Birmingham, UK

${ }^{2}$ Institute of Applied Health Research, University of Birmingham, Birmingham, UK ${ }^{3}$ National Institute for Health Research Applied Research Centre West Midlands, University of Birmingham, Birmingham, UK

${ }^{4}$ NIHR Birmingham Biomedical Research Centre, University of Birmingham, University of Birmingham, Birmingham, UK

${ }^{5}$ University Hospitals Birmingham NHS Foundation Trust, Birmingham, UK

${ }^{6}$ University of Warwick Warwick Medical School, Coventry, UK

${ }^{7}$ Birmingham Health Partners Centre for Regulatory Science and Innovation, Birmingham, UK

${ }^{8}$ Department of Social Work and Social Care, University of Birmingham, Birmingham, UK

${ }^{9}$ National Institute for Health Research Surgical Reconstruction and Microbiology Research Centre, Birmingham, UK

Twitter Sarah E Hughes @SarahHughesSLT, Samantha Cruz Rivera @samsamcr, Christel McMullan @christel_uob and Grace M Turner @gracemturner

Acknowledgements The authors wish to thank Sue Bayliss, Information Specialist, University of Birmingham for her assistance in developing the search strategy.

Contributors SEH, MC, OLA, DSL, JG conceptualised the study and contributed to the study design. SEH and PC developed the search strategy. GMT, SCR, CM contributed to methods design. SEH drafted and edited the manuscript with MC, OLA, DSL, JG, PC, GMT, SCR, CM providing critical revisions to the manuscript and supplementary material. The final version was read and approved by all authors (SEH, MC, OLA, DSL, JG, PC, GMT, SCR, CM).

Funding This research is funded by the National Institute for Health Research (NIHR) Applied Research Collaboration (ARC) West Midlands. The views expressed are those of the author(s) and not necessarily those of the NIHR or the Department of Health and Social Care.

Competing interests SEH is supported by the National Institute of Health Research (NIHR) Applied Research Centre (ARC), West Midlands. SH is company director of Narra Consulting Limited and undertakes consultancy work for Cochlear Ltd. $\mathrm{MC}$ is a National Institute for Health Research (NIHR) Senior Investigator and receives funding from the National Institute for Health Research (NIHR) Birmingham Biomedical Research Centre, the NIHR Surgical Reconstruction and Microbiology
Research Centre and NIHR ARC West Midlands at the University of Birmingham and University Hospitals Birmingham NHS Foundation Trust, Health Data Research UK, Innovate UK (part of UK Research and Innovation), Macmillan Cancer Support, UCB Pharma MC has received personal fees from Astellas, Takeda, Merck, Daiichi Sankyo, Glaukos, GSK and the Patient-Centered Outcomes Research Institute (PCORI) outside the submitted work. OLA is supported by the National Institute of Health Research (NIHR) Birmingham Biomedical Research Centre (BRC), Birmingham, West Midlands. OLA also receives funding from the Health Foundation and has received personal fees from Gilead Sciences. DL is supported by the NIHR ARC West Midlands and the NIHR Community Healthcare MedTech and IVD Cooperative, hosted by Oxford Health NHS Foundation Trust. JG is a non-executive director of University Hospitals Birmingham, adjunct professor at Curtin University, member of the leadership team for an ESRC large grant on sustainable adult social care, and member of the expert advisory group for West Midlands ARC. PC, GT, SCR, and $\mathrm{CM}$ have no conflicts of interest to declare.

Patient consent for publication Not required.

Provenance and peer review Not commissioned; externally peer reviewed.

Supplemental material This content has been supplied by the author(s). It has not been vetted by BMJ Publishing Group Limited (BMJ) and may not have been peer-reviewed. Any opinions or recommendations discussed are solely those of the author(s) and are not endorsed by BMJ. BMJ disclaims all liability and responsibility arising from any reliance placed on the content. Where the content includes any translated material, BMJ does not warrant the accuracy and reliability of the translations (including but not limited to local regulations, clinical guidelines, terminology, drug names and drug dosages), and is not responsible for any error and/or omissions arising from translation and adaptation or otherwise.

Open access This is an open access article distributed in accordance with the Creative Commons Attribution 4.0 Unported (CC BY 4.0) license, which permits others to copy, redistribute, remix, transform and build upon this work for any purpose, provided the original work is properly cited, a link to the licence is given, and indication of whether changes were made. See: https://creativecommons.org/ licenses/by/4.0/.

\section{ORCID iDs}

Sarah E Hughes http://orcid.org/0000-0001-5656-1198

Samantha Cruz Rivera http://orcid.org/0000-0002-1566-6804

Christel McMullan http://orcid.org/0000-0002-0878-1513

Melanie Calvert http://orcid.org/0000-0002-1856-837X

\section{REFERENCES}

1 Goodwin N, Curry N, Naylor C. Managing people with long term conditions. London: The King's Fund, 2010.

2 Vogeli C, Shields AE, Lee TA, et al. Multiple chronic conditions: prevalence, health consequences, and implications for quality, care management, and costs. J Gen Intern Med 2007;22:391-5.

3 Barnett K, Mercer SW, Norbury M, et al. Epidemiology of multimorbidity and implications for health care, research, and medical education: a cross-sectional study. Lancet 2012;380:37-43.

4 Glasby J. The Holy Grail of health and social care integration. BMJ 2017;356:j801.

5 FDA. Principles for selecting, developing, modifying, and adapting PatientReported outcome instruments for use in medical device evaluation: draft guidance for industry and food and drug administration staff, and other stakeholders 2020 (accessed 31 August 2020).

6 FDA. Guidance for industry: patient-reported outcome measures: use in medicinal product development to support labelling claims 2009.

7 Wilson IB, Cleary PD. Linking clinical variables with health-related quality of life. A conceptual model of patient outcomes. JAMA 1995;273:59-65.

8 Calvert M, Kyte D, Price G, et al. Maximising the impact of patient reported outcome assessment for patients and society. BMJ 2019;364:k5267.

9 Kyte D, Duffy H, Fletcher B, et al. Systematic evaluation of the patient-reported outcome (pro) content of clinical trial protocols. PLoS One 2014;9:e110229-e29.

10 FDA. Principles for selecting, developing, modifying, and adapting patient-reported outcome instruments for use in medical device evaluation: draft guidance for industry and food and drug administration staff, and other stakeholders: US food and drug administration 2020 . 
11 Murphy M, Hollinghurst S, Salisbury C. Identification, description and appraisal of generic PROMs for primary care: a systematic review. BMC Fam Pract 2018;19:41.

12 Schick-Makaroff K, Karimi-Dehkordi M, Cuthbertson L, et al. Using Patient- and Family-Reported outcome and experience measures across transitions of care for frail older adults living at home: a MetaNarrative synthesis. Gerontologist 2020. doi:10.1093/geront/gnz162. [Epub ahead of print: 16 Jan 2020].

13 Kearns T, Cornally N, Molloy W. Patient reported outcome measures of quality of end-of-life care: a systematic review. Maturitas 2017;96:16-25.

14 Johnston B, Flemming K, Narayanasamy MJ, et al. Patient reported outcome measures for measuring dignity in palliative and end of life care: a scoping review. BMC Health Serv Res 2017;17:574.

15 Ayton DR, Gardam ML, Pritchard EK, et al. Patient-Reported outcome measures to inform care of people with Dementia-A systematic scoping review. Gerontologist 2020;8.

16 Forestier B, Anthoine E, Reguiai Z, et al. A systematic review of dimensions evaluating patient experience in chronic illness. Health Qual Life Outcomes 2019;17:19

17 UK Government. The health and social care act 2008 (regulated activities) regulations 20142014.

18 Malley J, Netten A. Measuring outcomes of social care. Research, Policy, and Planning 2009;27:85-96.

19 Hackert MQN, van Exel J, Brouwer WBF. Does the ICECAP-O cover the physical, mental and social functioning of older people in the UK? Qual Life Res 2019;28:761-70.

20 Makai P, Brouwer WBF, Koopmanschap MA, et al. Quality of life instruments for economic evaluations in health and social care for older people: a systematic review. Soc Sci Med 2014;102:83-93.

21 Fund TKs. How to develop evidence-based solutions to improve patient journeys: The King's Fund 2020.

22 National collaboration for integrated care and support. integrated care and support 2013.

23 Greenhalgh T, Koh GCH, Car J. Covid-19: a remote assessment in primary care. BMJ 2020;368:m1182.

24 Aiyegbusi OL, Calvert MJ. Patient-Reported outcomes: central to the management of COVID-19. Lancet 2020;396:531.

25 Taylor PC. Adopting pros in virtual and outpatient management of RA. Nat Rev Rheumatol 2020;16:477-8.

26 Humphries R. Integrated health and social care in England--Progress and prospects. Health Policy 2015;119:856-9.

27 Glasby J. Understanding health and social care. Revised, 3rd ed. Bristol University Press, 2017.

28 Peterson J, Pearce PF, Ferguson LA, et al. Understanding scoping reviews: definition, purpose, and process. J Am Assoc Nurse Pract 2017;29:12-16.
29 Munn Z, Peters MDJ, Stern C, et al. Systematic review or scoping review? guidance for authors when choosing between a systematic or scoping review approach. BMC Med Res Methodol 2018;18:143.

30 Arksey H, O'Malley L. Scoping studies: towards a methodological framework. Int J Soc Res Methodol 2005;8:19-32.

31 Peters M, Godfrey C, Mclnerney P. Chapter 11: Scoping Reviews (2020 Version). In: Aromataris E, Munn Z, eds. JBI manual for evidence synthesis. JBI, 2020

32 Moher D, Shamseer L, Clarke M, et al. Preferred reporting items for systematic review and meta-analysis protocols (PRISMA-P) 2015 statement. Syst Rev 2015;4:1.

33 Tricco AC, Lillie E, Zarin W, et al. PRISMA extension for scoping reviews (PRISMA-ScR): checklist and explanation. Ann Intern Med 2018;169:467-73.

34 Think local act personal. TLAP care and support jargon buster. Available: https://www.thinklocalactpersonal.org.uk/Browse/ Informationandadvice/CareandSupportJargonBuster/ [Accessed 27 Jan 2021].

35 Harris J, White V. Oxford dictionary of social work and social care. 2nd edn. Oxford: Ocford University Press, 2018.

36 Bramer WM, Rethlefsen ML, Kleijnen J, et al. Optimal database combinations for literature searches in systematic reviews: a prospective exploratory study. Syst Rev 2017;6:245

37 Godin K, Stapleton J, Kirkpatrick SI, et al. Applying systematic review search methods to the grey literature: a case study examining guidelines for school-based breakfast programs in Canada. Syst Rev 2015;4:138

38 Devlin NJ, Appleby J. Getting the most out of PROMs. London: The King's Fund, 2010.

39 Equity and Excellence. Liberating the NHS. in: health do, ED. London: HMSO, 2010.

40 Casp checklists, 2020. Available: https://casp-uk.net/casp-toolschecklists/ [Accessed 28 Aug 2020].

41 Tyndall J. ACCODS checklist for appraising grey literature, 2010. Available: https://dspace.flinders.edu.au/jspui/bitstream/2328/3326/ 4/AACODS_Checklist.pdf [Accessed 20 September 2020].

42 Shrout PE, Fleiss JL. Intraclass correlations: uses in assessing rater reliability. Psychol Bull 1979;86:420-8.

43 Cohen J. A coefficient of agreement for nominal scales. Educ Psychol Meas 1960;20:37-46.

44 Gale NK, Heath G, Cameron E, et al. Using the framework method for the analysis of qualitative data in multi-disciplinary health research. BMC Med Res Methodol 2013;13:117.

45 Ritchie J, Spence L. Qualitative data analysis for applied policy research. In: Bryman A, Burgess RG, eds. Analyzing qualitative data. New York: Routledge, 1994.

46 Staniszewska S, Brett J, Simera I, et al. GRIPP2 reporting checklists: tools to improve reporting of patient and public involvement in research. Res Involv Engagem 2017;3:13. 